\title{
Estado nutricio, presión arterial y su relación con la disgeusia en adultos mayores con hipertensión arterial sistémica de Aguascalientes, México
}

\author{
López-Gutiérrez Norma Patricia*, Contreras-Salazar María José*, Ramírez Orozco Ricardo Ernesto*
}

\section{Resumen}

- Introducción: el envejecimiento celular involucra una serie de alte-

- raciones en el organismo, entre ellas la distorsión en el sentido del

- gusto o disgeusia, que puede tener un impacto negativo en la salud

- del adulto mayor, por ejemplo, en la hipertensión arterial sistémica

- (HAS). Se evaluó la relación del estado nutrición con el control de

- los niveles de presión arterial y disgeusia en adultos mayores con

- HAS. Materiales y métodos: se incluyeron 72 adultos mayores con

- HAS evaluando detección y percepción del sabor salado mediante

- pruebas de feniltiocarbamida y soluciones salinas y su relación con el

- estado nutricio. Resultados: en el $76.4 \%$ de los pacientes con hiper-

- tensos evaluados, padecen una alteración gustativa de percepción

- de la solución salina ( $p=0.008)$. Se encontró que los pacientes con

- mayor presión arterial sistólica, requieren una mayor concentración

- salina para su detección ( $p=0.006$ ). Conclusión: la disminución de

- la percepción del sabor salado, puede contribuir al descontrol de los

- niveles de presión arterial sistólica. LUX MÉDICA AÑO 15, NÚMERO 43,

- ENERO-ABRIL 2020.PP 25-33

Palabras claves: detección del gusto, sabor salado, feniltiocarbamida, obesidad, presión arterial.

\section{Abstract}

Introduction: Cellular aging involves a series of alterations in the body, including distortion in the sense of taste or dysgeusia, which can have a negative impact on the health of the elderly, for example, in systemic hypertension (SH). The relationship of the nutritional status with blood pressure control and dysgeusia in the elderly with SH was evaluated. Methods: Seventy-two elderly patients with SH were included, evaluating the detection and perception of salty taste using phenylthiocarbamide and saline solutions and their relationship with nutritional status. Results: $76.4 \%$ of patients with hypertension experienced a taste distortion to saline solution ( $p=0.008$ ). It was found that patients with higher systolic blood pressure require a higher saline concentration for their detection $(p=0.006)$. Conclusions: Decrease in the perception of salty taste can contribute to the lack of control of systolic blood pressure levels. LUX MÉDICA AÑO 15, NÚMERO 43, ENERO-ABRIL 2020. PP 25-33

Keywords: taste detection, salty taste, phenylthiocarbamide, obesity, blood pressure

\section{Introducción}

Actualmente en México, el $25.5 \%$ de la población adulta padece hipertensión arterial sistémica (HAS), de la cual el $49.3 \%$ son adultos mayores. El número de personas con hipertensión ha ido

* Licenciada en Nutrición, egresada de la Universidad Autónoma de Aguascalientes, correo electrónico: lopez.paty91@yahoo.com Estudiante de la maestría en Ciencias Médicas por parte de la universidad de Guanajuato, correo: maryjo.cs@hotmail.com

Fecha de recibido 11 de octubre 2019

Fecha de aceptación: 19 de noviembre 2019

Correspondencia: Dr. Ricardo Ernesto Ramírez Orozco. Departamento de Nutrición. Edificio 105, Centro de Ciencias de la Salud. Ciudad Universitaria. Universidad Autónoma de Aguascalientes. Avenida Universidad 940. Código postal 20131. Aguascalientes, Ags., México Teléfono 9107400 extensión 53355. Correo electrónico dcmrero@gmail.com. 
en aumento en los últimos años y la mayor parte desconoce su condición. ${ }^{1}$ El adulto mayor tiene más susceptibilidad de desarrollar enfermedades crónico-degenerativas como HAS a causa del proceso de envejecimiento, en el cual ocurren cambios que afectan de manera importante la funcionalidad de su organismo. Las pérdidas sensoriales en diferentes grados de severidad, son uno de los principales efectos que ocurren en la senectud. ${ }^{2,3}$

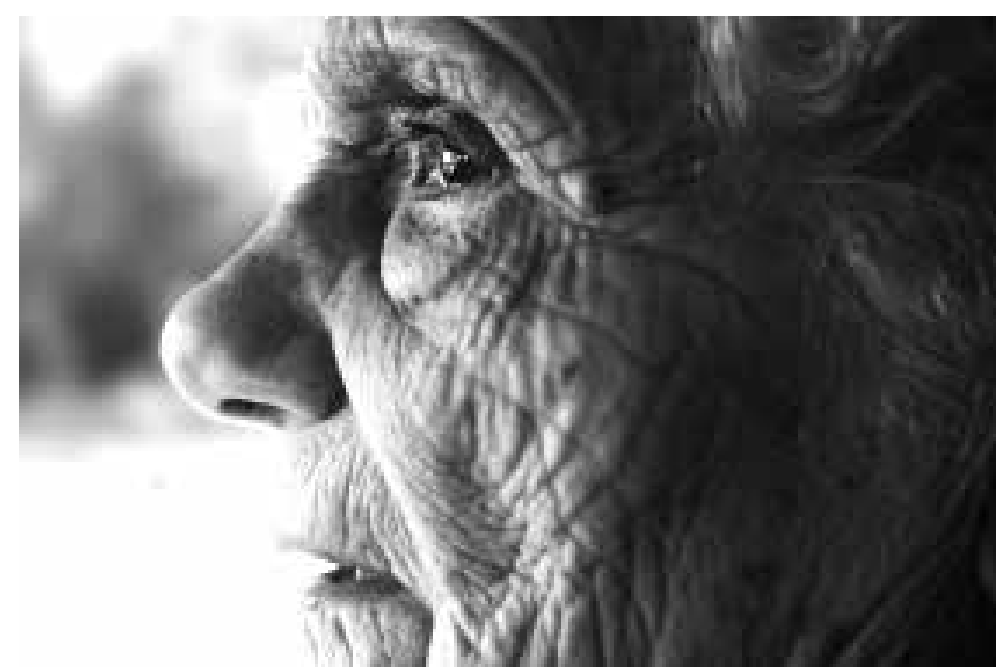

Figura 1. Fuente:https://pixabay.com/es/adulto-mayor-viejo-675338/.

La valoración de las alteraciones del gusto se realizan por medio de pruebas sensoriales gustativas, donde los individuos responden a estímulos; entre ellas está la prueba de solución salina a diferentes concentraciones para identificar el umbral sensorial. ${ }^{4}$ La prueba de feniltiocarbamida (PTC) es empleada para la detección de alteraciones gustativas, la cual permite clasificar a los individuos en grupo de disgeusia, hipogeusia e hipergeusia. ${ }^{5}$ La alteración del gusto puede ser causada por algunas enfermedades, como el sobrepeso, obesidad y diabetes mellitus; también el uso de algunos fármacos, como los antihipertensivos, pueden ser un desencadenante de esta condición, ${ }^{6}$ que ocasionan una disminución de la sensibilidad y percepción gustativa, donde la percepción de insipidez de las comidas puede causar un exceso en el consumo de sal en los alimentos $y$, por consiguiente, aumentar el consumo de sodio, promoviendo un pobre control de los niveles de presión arterial y la aparición de complicaciones. ${ }^{7,8}$ El objetivo de este estudio fue describir la prevalencia y relación entre la alteración de la percepción del sabor salado y el control de los niveles de presión arterial en el adulto mayor con hipertensión que residen en estancias para adultos mayores en Aguascalientes, México. 


\section{Material y métodos}

a Participantes: se seleccionó una muestra total de 72 adultos mayores $a \geq 60$ años (36 hombres y 36 mujeres), residentes de la Estancia de Día "Casa Del Abuelo" y del Centro de Rehabilitación Integral y Social del DIF estatal de Aguascalientes, México, con diagnóstico de hipertensión arterial; fueron excluidos pacientes con tratamiento previo de radioterapia o quimioterapia o con enfermedades que podían alterar la percepción del gusto; cada persona firmó consentimiento informado para participar en el estudio, el proyecto fue sometido y aprobado por el Comité de Ética del Departamento de Nutrición y Cultura Física de la Universidad Autónoma de Aguascalientes. Los participantes fueron reclutados por disponibilidad, considerando el tamaño de muestra mínimo de acuerdo al estudio realizado por Piovesana y cols. ${ }^{9}$

b Pruebas de sensibilidad gustativa: prueba PTC (feniltiocarbamida) y prueba de solución salina. Los criterios de alteración del gusto van de acuerdo a la siguiente clasificación: disgeusia, hipogeusia e hipergeusia. A cada participante se le colocó una tira reactiva de PTC (5 $\mu \mathrm{g} /$ tira) marca Precisión Laboratories ${ }^{\circledR}$ en la punta de la lengua presionando contra el paladar durante un tiempo mínimo de cinco segundos para identificar el sabor de dicha prueba; si el paciente identificaba un sabor amargo intenso, se clasificaba en el grupo de hipergeusia; la detección de un sabor ligero, casi imperceptible, fue clasificado como hipogeusia; no detectar el sabor fue clasificado como disgeusia. ${ }^{10}$ Por otro lado, se prepararon una serie de muestras representativas de solución salina a concentraciones diferentes de $\mathrm{NaCl}(0.25 \mathrm{~g} / \mathrm{L}, 0.5 \mathrm{~g} / \mathrm{L}$ y $0.75 \mathrm{~g} / \mathrm{L})$ de acuerdo a la técnica escalonada de Cornsweet. ${ }^{411}$ Los participantes bebieron dos vasos, uno con agua y otro con la solución salina, de menor a mayor concentración hasta la identificación del umbral de detección y reconocimiento de sabor salado. Si no se detectaba el sabor salino se daba a probar la siguiente concentración, con previo lavado bucal con enjuague y agua entre cada prueba. Cada concentración se hizo por duplicado. ${ }^{12}$

c Evaluación del estado nutricio y medición de la presión arterial: se evaluó la composición corporal por medio de la báscula TANITA ${ }^{\circledR}$ modelo: BC-148. Se obtuvo el peso, talla, circunferencia de cintura, porcentaje de grasa y kilogramos de músculo. El IMC fue establecidos considerando los siguientes valores: bajo peso $\left(<22 \mathrm{~kg} / \mathrm{m}^{2}\right)$; peso normal $\left(22-27 \mathrm{~kg} / \mathrm{m}^{2}\right)$; sobrepeso $(27.1-30 \mathrm{~kg} /$ $\mathrm{m}^{2}$ ); obesidad $\left(>30 \mathrm{~kg} / \mathrm{m}^{2}\right.$ ) de acuerdo a la Guía de Práctica Clínica: Evaluación y seguimiento nutricional del adulto mayor en el primer nivel de atención (CNETS, 2018). ${ }^{13}$ Para clasificar el porcentaje de grasa corporal se empleó el sistema de clasificación propuesto por Lohman, considerando alto cuando la grasa corporal es $>25.0 \%$ en hombres y de $>32.0 \%$ en mujeres. ${ }^{14}$ Para la determinación de la presión arterial, cada paciente guardó reposo durante cinco minutos. Se evaluaron los niveles de presión arterial con un esfigmomanómetro de mercurio. Se realizaron dos mediciones separadas por cinco minutos. El valor final asignado a cada sujeto fue la media de las dos mediciones. ${ }^{15}$

Análisis estadístico: se utilizaron medias y desviación estándar para la representación de los datos en la estadística descriptiva. La normalidad se evaluó con la prueba de Kolmogorov-Smirnov. Se aplicó T de Student y ANOVA no paramétrico (Kruskal-Wallis) y el post hoc Dunn's para analizar diferencias entre grupos. Mediante la correlación de Pearson y la prueba de $\chi^{2}$ se evaluaron asociaciones entre variables antropométricas y la presión arterial y concen- 
tración de solución salina. Se consideró una $p<0.05$ para el nivel de significan- cia, los datos fueron analizados en el software SPSS versión 20.

\section{| | | | | | | | | | | | | | | | | | | | | | | | | | | | | | | | | | | | | | | | | | | | | | | | | | | | | | | | | | | | | | | | | | | | | | | | | | | | | | | | | | | | | | | | | | | | | | | | | | | | | | | | | | | | | | |}

\section{Resultados}

\section{Características descriptivas de la población estudiada}

Se evaluaron un total de 72 adultos mayores con HAS, con edad promedio de $75.8 \pm 8.1$,

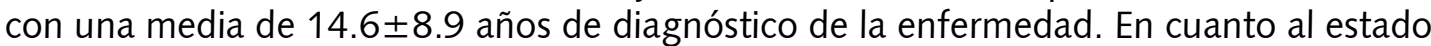
nutricio, el $6.9 \%$ presentó bajo peso; el $47.2 \%$, normopeso; $19.4 \%$, sobrepeso; y el $26.4 \%$, obesidad. En relación con los niveles de presión arterial, los hombres presentaron niveles mayores $(78.2 \pm 7.5 \mathrm{mmHg}$ ) en la presión arterial diastólica en comparación con las mujeres $(p=0.006)$; por otro lado, el número de fármacos consumidos en el grupo de mujeres fue de $3.3 \pm 1.2$, siendo mayor en comparación con los hombres $(p=0.033)$. Se pueden mostrar el resto de las características estudiadas en la tabla 1.

\section{Tabla |}

\section{Características generales de la población estudiada de acuerdo con el sexo}

\begin{tabular}{|lllll|}
\hline Variable & $\begin{array}{l}\text { Hombre } \\
(\mathrm{n}=36)\end{array}$ & $\begin{array}{l}\text { Mujer } \\
(\mathrm{n}=36)\end{array}$ & $\mathrm{F}$ & $\mathrm{p}$ \\
\hline Edad & $74.3 \pm 8.7$ & $77.2 \pm 7.2$ & 2.0 & 0.121 \\
\hline Peso $(\mathrm{kg})$ & $75.0 \pm 15.2$ & $64.3 \pm 11.4$ & 1.8 & $\mathbf{0 . 0 0 1}$ \\
\hline Estatura $(\mathrm{m})$ & $1.6 \pm 0.0$ & $1.5 \pm 0.0$ & 0.1 & 0.001 \\
\hline IMC $(\mathrm{kg} / \mathrm{m} 2)$ & $27.1 \pm 3.9$ & $28.0 \pm 5.1$ & 1.6 & 0.387 \\
\hline Masa grasa $(\%)$ & $28.4 \pm 6.8$ & $35.5 \pm 6.1$ & 1.4 & 0.001 \\
\hline Masa muscular $(\mathrm{kg})$ & $40.9 \pm 11.8$ & $39.0 \pm 5.5$ & 27.3 & 0.385 \\
\hline Circunferencia de cintura $(\mathrm{cm})$ & $101.9 \pm 11.4$ & $99.0 \pm 10.2$ & 0.6 & 0.272 \\
\hline PAS (mmHg) & $137.9 \pm 13.2$ & $131.5 \pm 16.5$ & 2.9 & 0.075 \\
\hline PAD (mmHg) & $78.2 \pm 7.5$ & $73.5 \pm 6.4$ & 0.5 & 0.006 \\
\hline Fármacos consumidos (n) & $2.6 \pm 1.3$ & $3.3 \pm 1.2$ & 0.2 & 0.033 \\
\hline Tiempo con HAS (años) & $13.4 \pm 8.3$ & $15.4 \pm 9.3$ & 0.1 & 0.418 \\
\hline
\end{tabular}

Datos mostrados en media y desviación estándar. Prueba utilizada: T de student; $p<0.05$. PAS: presión arterial sistólica, PAD: presión arterial diastólica.

\section{El estado nutricio y la percepción del sentido del gusto}

Por medio de la prueba PTC se observó una prevalencia alta en la alteración del sabor: disgeusia en el $54.2 \%$ de la población, $22.2 \%$ con hipogeusia y $23.6 \%$ con hipergeusia; no obstante, no fueron encontradas diferencias entre la percepción del sabor y el IMC de los pacientes. Por otro lado, la percepción del sabor salino fue adecuada entre los pacientes con hipergeusia en comparación a los otros grupos $(p=0.008)$, de acuerdo a lo mostrado en la tabla 2 y figura 2 . En el grupo con IMC elevado se encontraron mayores niveles de presión arterial diastólica y mayor consumo de medicamentos, $(p=0.013$ y $p=0.032$ respectivamente) figura 2 . 


\section{Tabla 2}

Diferencia entre grupos de clasificación de percepción del sabor y detección

\begin{tabular}{|lcccccc|}
\hline Variable & Disgeusia $(\mathrm{n}=39)$ & Hipogeusia $(\mathrm{n}=16)$ & Hipergeusia $(\mathrm{n}=17)$ & $\mathrm{H}$ & $\mathrm{p}$ & Post Hoc \\
\hline Solución Detectable $(\mathrm{g} / \mathrm{L})$ & $0.6 \pm 0.1$ & $0.5 \pm 0.1$ & $0.4 \pm 0.2$ & 9.6 & 0.008 & 0.049 \\
\hline Fármacos consumidos $(\mathrm{n})$ & $2.7 \pm 1.2$ & $3.3 \pm 1.0$ & $3.0 \pm 1.7$ & 2.5 & 0.285 & 0.350 \\
\hline Diagnóstico de HAS (años) & $16.1 \pm 8.9$ & $15.0 \pm 10.6$ & $11.0 \pm 6.4$ & 2.8 & 0.237 & 0.239 \\
\hline
\end{tabular}

Datos mostrados en media y desviación estándar. (n=72). Prueba utilizada: Kruskal-Wallis y post hoc Dunn's. $p<0.05$.
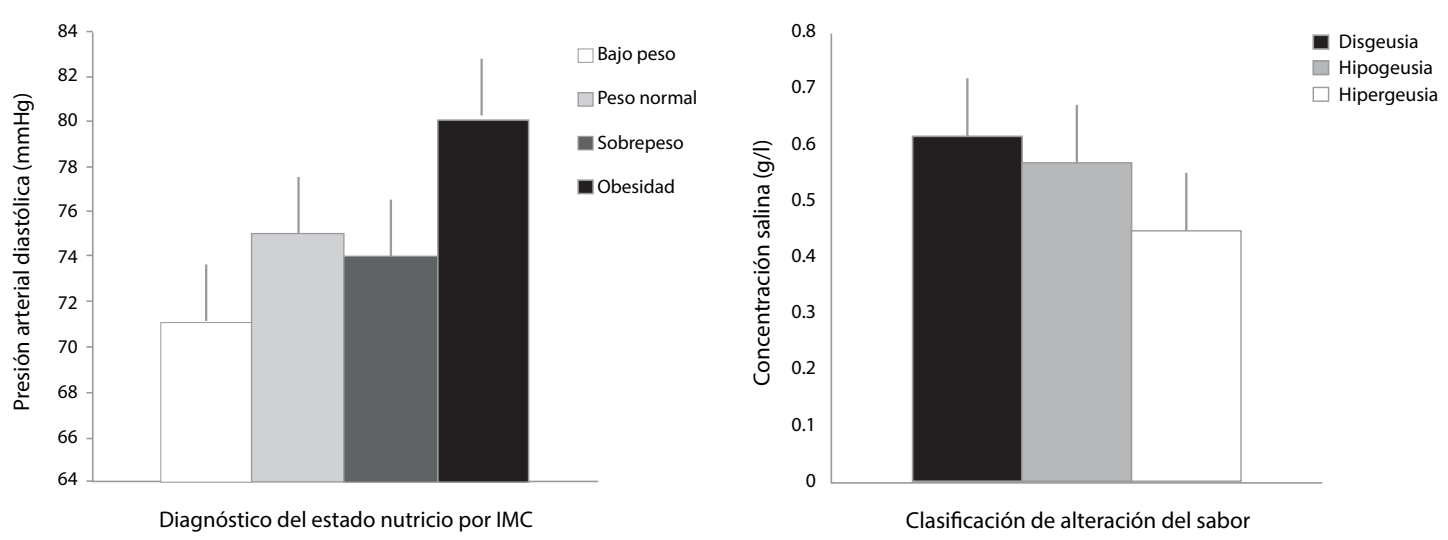

Figura 2. a) Niveles de presión arterial diastólica de acuerdo al índice de masa corporal.b) Detección del sabor salado de acuerdo a la clasificación de alteración del gusto.

Finalmente, los adultos mayores con sobrepeso son quienes tienen mayor tiempo con diagnóstico de hipertensión (Tabla 3).

\section{Tabla 3}

Diferencia entre grupos de acuerdo a clasificación de IMC

\begin{tabular}{|llllllll|}
\hline Variable & $\begin{array}{l}\text { Bajo peso } \\
(\mathrm{n}=5)\end{array}$ & $\begin{array}{l}\text { Peso normal } \\
(\mathrm{n}=34)\end{array}$ & $\begin{array}{l}\text { Sobrepeso } \\
(\mathrm{n}=14)\end{array}$ & $\begin{array}{l}\text { Obesidad } \\
(\mathrm{n}=19)\end{array}$ & $\mathrm{H}$ & $\mathrm{p}$ & Post Hoc \\
\hline PAS (mmHg) & $134 \pm 15.7$ & $133 \pm 14.5$ & $134 \pm 12.8$ & $137 \pm 18.3$ & 2.0 & 0.57 & 0.90 \\
\hline PAD (mmHg) & $71 \pm 3.8$ & $75 \pm 7.1$ & $74 \pm 6.1$ & $80 \pm 7.9$ & 10.7 & 0.01 & 0.04 \\
\hline Solución detectable (g/L) & $0.6 \pm 0.1$ & $0.5 \pm 0.1$ & $0.5 \pm 0.1$ & $0.6 \pm 0.2$ & 1.6 & 0.65 & 0.89 \\
\hline Fármacos consumidos ( $\mathrm{n})$ & $1.6 \pm 0.5$ & $3.0 \pm 1.3$ & $2.5 \pm 1.3$ & $2.8 \pm 1.3$ & 8.8 & 0.03 & 0.04 \\
\hline Diagnóstico de HAS (años) & $7.6 \pm 5.0$ & $13.5 \pm 7.0$ & $21.2 \pm 10.1$ & $14.6 \pm 8.9$ & 8.9 & 0.03 & 0.04 \\
\hline
\end{tabular}

Datos mostrados en media y desviación estándar. (n=72). Prueba utilizada: Kruskal-Wallis y post hoc

Dunn's. $p<0.05$. 


\section{Detección y percepción del sabor y su asociación con la HAS en el adulto mayor}

Se encontró hipogeusia en el $70.8 \%$ de los adultos estudiados ya que no detectaron el sabor salado de una solución salina al $0.25 \mathrm{~g} / \mathrm{L} .\left(\chi^{2}=8.5, \mathrm{p}=0.014\right)$; mientras que el $40.2 \%$ (disgeusia e hipogeusia) tampoco detectaron el sabor salado $\left(\chi^{2}=6.5, p=\right.$ 0.038), utilizando una solución salina al $0.50 \mathrm{~g} / \mathrm{L}$.

Existe una asociación positiva entre la concentración de salinidad en solución y la masa muscular ( $r=0.302, p=0.010)$, observando que una mayor masa muscular presente, mayor es la concentración en la prueba de detección de las soluciones. En cuanto a la asociación entre la presión arterial y la solución detectable $(R=0.320, p=0.006)$, una mayor presión arterial sistólica condicionará una mayor concentración de salinidad para su detección. Finalmente, un incremento en el número de fármacos consumidos con el tiempo de evolución de la enfermedad $(R=0.307, p=0.021)$, todas la variables pueden ser revisadas en la tabla 4.

\section{Tabla 4}

\section{Correlación entre variables antropométricas y percepción del sabor y años de evolución con HAS}

\begin{tabular}{|c|c|c|c|}
\hline Variable & Variable regresora & $\mathbf{R}$ & $\mathbf{p}$ \\
\hline Peso (kg) & & -0.032 & 0.791 \\
\hline IMC (kg/m2) & & 0.040 & 0.740 \\
\hline Masa grasa (\%) & & 0.053 & 0.656 \\
\hline Masa muscular (kg) & Solución detectable $(\mathrm{g} / \mathrm{L})$ & 0.302 & 0.010 \\
\hline Circunferencia de cintura $(\mathrm{cm})$ & & -0.023 & 0.845 \\
\hline Presión arterial sistólica (mmHg) & & 0.320 & 0.006 \\
\hline Presión arterial diastólica (mmHg) & & 0.136 & 0.254 \\
\hline Fármacos consumidos $(\mathrm{n})$ & Diagnóstico de HAS (años) & 0.307 & 0.021 \\
\hline
\end{tabular}

Prueba utilizada: correlación de Pearson, $p<0.05$.

\section{||||||||||||||||||||||||||||||||||||||||||||||||||||||||||||||||||||||||||||||||||||||||||||||||||||||||||||||||||||||}

\section{Discusión}

La relación entre la alteración de la percepción del sabor y el control de los niveles de presión arterial en el adulto mayor con hipertensión denota importancia clínica, ya que esto puede determinar el apego al tratamiento nutricio y el retardo de la aparición y desarrollo de complicaciones de la hipertensión arterial. Nuestra población de estudio presentó una prevalencia importante de adultos mayores con sobrepeso u obesidad (45.8\%), lo cual es congruente con la Encuesta de Salud y Nutrición ENSANUT $2016^{1}$ que describe una prevalencia de hasta $69.4 \%$ de población adulta ma- yor. Esta condición favorece la aparición de enfermedades crónico-degenerativas y sus complicaciones son las principales causas de mortalidad en nuestro país.

Con respecto a la población con obesidad y un aumento en la presión arterial diastólica, Campos y cols. ${ }^{16}$ observaron la misma relación en la población mexicana donde exponen que la prevalencia de hipertensión fue 1.3 veces más alta en adultos con obesidad; sin embargo, no encuentran diferencia entre presión arterial sistólica y diastólica, probablemente debido a los años de diagnóstico con la enfermedad y del control por medicamento, dieta y 
ejercicio. Por otro lado, Landsberg y cols. ${ }^{17}$ nos hablan de la hipertensión relacionada con la obesidad, reconocida como factor de riesgo para la enfermedad cardiovascular en ambos sexos. Se han hipotetizado mecanismos por el cual la obesidad contribuye a la elevación de la presión arterial, tal es el ejemplo del sistema eicosanoide, ${ }^{18}$ reportan resistencia a la insulina en presencia de obesidad, la cual fue asociada a una mayor excreción urinaria de 20-HETE (ácido hidroxieicosatetraenoico), un vasoconstrictor potente de la musculatura lisa, haciendo que progrese la hipertensión en la obesidad. ${ }^{18}$

Los resultados de este estudio concuerdan con los resultados de Campos y cols. ${ }^{16}$ donde describen niveles mayores de presión arterial en el hombre en comparación con la mujer en la adultez mayor, una diferencia promedio de 3-7 mmHg; sin embargo, conforme aumenta la edad en la tercera edad, la presión arterial sistólica particularmente se incrementa en la mujer. Autores como Campos y cols. ${ }^{18}$ y Moulana y cols. ${ }^{18}$ explican la posibilidad de que la presión arterial sea regulada de diversas maneras en cada sexo; es decir, probablemente las diferentes hormonas como los andrógenos y estrógenos tengan un papel en la regulación de la presión arterial; sin embargo, exponen que de igual manera, la obesidad es un factor de riesgo que incrementa la posibilidad de hipertensión arterial sistémica en ambos sexos.

El control del peso corporal y la disminución de la circunferencia de cintura mejoran los niveles de presión arterial; como indican nuestros resultados, un aumento en éstos condiciona el incremento de la presión arterial diastólica. Peterson y cols. ${ }^{19}$ afirman que un aumento de peso y por ende del IMC por arriba de $30 \mathrm{~kg} / \mathrm{m}^{2}$ aumenta la oxidación plasmática de HDL y los niveles de isoprostano, a su vez incrementando 20-HETE y angiotensina II, siendo parte de las probables explicaciones por las cuales el estado nutricio marca una importancia en la hipertensión o aparición de ésta. ${ }^{19}$ El $76.4 \%$ de la población padecía alteración en el sentido del gusto, corroborando lo descrito por Muñoz y cols. ${ }^{20}$ que evaluaron aspectos nutricionales y su relación con anomalías en el sentido del gusto, encontraron alteraciones gustativas en el $73,3 \%$ de adultos mayores, confirmando los cambios fisiológicos atribuibles al envejecimiento celular. ${ }^{20}$ Nuestros resultados concuerdan con lo establecido por el diagnóstico de disgeusia, es decir, en la muestra estudiada se requirió una mayor concentración de las soluciones salinas para la detección; diversos factores pueden estar implicados en la aparición de esta alteración, ya que la mayor parte de los participantes consumían numerosos medicamentos. Lee y cols. ${ }^{21}$ nos hablan sobre la excreción en saliva de una gran parte de medicamentos por medio de transporte pasivo o difusión, que pueden afectar el sentido del gusto por diversos mecanismos, incluyendo interacción fármaco-receptor, esto alterará el potencial de propagación en las membranas celulares en neuronas aferentes y eferentes, además de alterar la función de neurotransmisores. ${ }^{21}$

Un resultado contrastante a lo esperado es la cantidad de masa muscular y su relación con la detección y percepción de la solución salina. Actualmente no existe información documentada sobre esta aseveración; sin embargo, podemos asumir que esto podría tratarse del aumento de masa muscular que se da en el sobrepeso y obesidad. Pese al incremento de masa grasa, éste va acompañado de una ganancia respectiva de masa muscular. No obstante, a pesar de que nuestra población son adultos mayores, esperaríamos la presencia de obesidad sarcopénica en esta población; pero Tallis y cols. ${ }^{22}$ nos hablan sobre los efectos agudos de la obesidad sobre la fuerza y potencia en el mantenimiento de la masa muscular, aunque este efecto se pierde de manera sinérgica con la obesidad y el envejecimiento, causando problemas 
de contractilidad y relajación. ${ }^{22}$ Este hallazgo nos lleva a pensar que el aumento de masa muscular enmascarado por el sobrepeso de los pacientes con HAS, hace que necesiten de una mayor concentración salina para la percepción del sabor, dando una respuesta probable a este evento, siendo la disgeusia o hipogeusia una aparición temprana de las complicaciones de la hipertensión arterial descontrolada por la presencia de obesidad.
Finalmente, la asociación entre el incremento de presión arterial sistólica y la cantidad necesaria para la percepción de la solución detectable, ha sido atribuido al efecto de fármacos antihipertensivos que afectan el apetito e impactan en la percepción del sabor a nivel del receptor TAS1R y TAS2R en población de adultos mayores ${ }^{23}$ pudiera explicar nuestros hallazgos sobre la hipogeusia y disgeusia entre la población adulto mayor con hipertensión arterial.

\section{Conclusiones}

El control metabólico mediante un estado de nutrición adecuado, permitirá un mantenimiento de niveles de presión arterial adecuados. La detección temprana de alteraciones en el gusto podrá determinar estrategias que aumenten la efectividad de los tratamientos antihipertensivos, mejorando la expectativa y calidad del adulto mayor con HAS. Añadir alimentos ricos en sodio, debido a una mala percepción del sabor salado condicionará la aparición temprana de complicaciones de la HAS, tales como: eventos vasculares cerebrales, complicaciones cardiacas, renales o del mismo endotelio, mermando incluso aún más el estado de salud.

Es necesario, ante el incremento de la prevalencia de la hipertensión arterial sistémica, implementar medidas preventivas de calidad. La restricción en la ingesta de sodio, el control de peso, el cambio de hábitos como la dieta y actividad física, además de pruebas para la determinación del sentido del gusto que permitan conocer la evolución de nuestra población con hipertensión arterial o con riesgo de desarrollarla.

\section{Bibliografía}

1 Hernández $M$, Rivera J, Shamah T, Cuevas L, Gómez LM, Gaona EB, et. al. (2016). Encuesta Nacional de Salud y Nutrición de Medio Camino 2016. Cuernavaca, Instituto Nacional de Salud Pública.

2 Kotchen TA, Vasculopatía Hipertensiva. En: Kasper D, Fauci A, Hauser S, Longo D, et al. (2016). Harrison principios de medicina interna. México, McGraw?Hill Interamericana, Vol 2. 19a ed; p 1611-1621.

3 Mahan LK, Escott-Stump S. (2013). Nutrición y dietoterapia de Krause. México, Mc Graw-Hill Interamericana; p. 444-448.

4 Vijande M, Málaga S, Argüelles J, Málaga I. Perillán C, Díaz JJ. (2005). Alteraciones de la sensibilidad gustativa a la salen hijos de hipertensos esenciales: Hipertens Riesgo Vasc; 22(5): 311-7.

5 Doty RL, De Fonte TP. (2016). Relationship of Phenylthiocarbamide (PTC): Taster Status to Olfactory and Gustatory Function in Patients with Chemosensory Disturbances, Chem Senses. 41 (8): 685-96.

6 Stewart JE, Seimon RV, Otto B, Keast RS, Clifton PM, Feinle Bisset C, (2011). Marked differences in gustatory and gastrointestinal sensitivity to oleic acid between lean and obese men, Am J Clin Nutr; 93(4):703-11.

7 Wasalathanthri S, Hettiarachchi P, Prathapan S, (2014). Sweet taste sensitivity in pre-diabetics, diabetics and normoglycemic controls: a comparative cross sectional study, BMC Endocr Disord, 14(1): 6-7.

8 Suliburska J, Duda G, Pupek-Musialik D, (2012). The influence of hypotensive drugs on the taste sensitivity in patients with primary hypertension, Acta Pol Pharm, 69(1):121-7.

9 Piovesana M, Sampaio K, Gallani MC. (2013). Association between Taste Sensitivity and Self-Reportedand: Objective Measures of Salt Intake among 
Hypertensive and Normotensive Individuals, ISRN Nutr, 301213:1-7

10 Preclaboratories.com [Internet]; EUA [Consultada 15 de febrero del 2018] Disponible en: https://preclaboratories.com/wp-content/uploads/2018/01/165PTC-Test-Paper-SDS.pdf.

11 Cornsweet TN. (1962). The staircase-method in psychophysics. Am J Psychol. 75:485-91.

12. Suarez C, Gil-Carcedo LM, Marco J, Medina JE, Ortega P, Trinidad J. (2008).Tratado de Otorrinologia y Cirugia de Cabeza y cuello, Buenos Aires; Editorial Medica Panamericana, $2^{\text {a }}$ Edición Tomo III, 2282 2284.

13 Centro Nacional de Excelencia Tecnológica en Salud [Internet]: Guía Práctica Clínica: Evaluación y seguimiento nutricional del adulto mayor en el Primer Nivel de Atención, [Citado el 15 de febrero del 2018]. Disponible en: http://www.cenetec.salud.gob. $\mathrm{mx} /$ descargas/gpc/CatalogoMaestro/095_GPC Evaycontrolnutic_eneladultomayor/nutricion_am_ rr_cenetec.pdf.

14 Aristizábal JC, Restrepo MT, Estrada A. (2007), Evaluación de la composición corporal de adultos sanos por antropometría e impedancia bioeléctrica. Biomedica. 27(2): 216-224.

15 Gómez A, Morales S, Álvarez CJ. (2016). Técnica para una correcta toma de la presión arterial en el paciente ambulatorio, Rev. Fac. Med. 59(3).

16 Campos C, Sala J, Vilariño O, Serra H, Del Sueldo M, Del Suelo ML. (2005), Capítulo II: Diferencias Relacionadas con el Sexo. En Serra C, Sala JP, Balestrini CE. Enfermedad Coronaria en la Mujer ¿Dónde están las diferencias?, [Internet]. Argentina, Instituto Modelo de Cardiología. [Citado el 15 de febrero del 2018] Disponible en: http://www.fac.org.ar/ccvc/ llave/libro01/cap02.pdf.
17 Landsberg L, Aronne LJ, Beilin LJ, Burke V, Igel LI, Lloyd-Jones D, Sowers J. (2013). Obesity-related hypertension: pathogenesis, cardiovascular risk, and treatment: a position paper of The Obesity Society and the American Society of Hypertension. J Clin Hypertens (Greenwich).15:14-33.

18 Laffer CL, Laniado-Schwartzman M, Nasjletti A, Elijovich F. (2004), 20-HETE and Circulating Insulin in Essential Hypertension With Obesity. Hypertension, 43:388-392.

19 Moulana M, Maranon RO. (2018). Regulation of blood pressure is influenced by gender: A study in obese Zucker rats. Life Sci. pii: S00243205(18)30466-1.

20 Peterson SJ, Vanella L, Gotlinger K, Jiang $H$, Singh SP, Sodhi K, Maher E, O'Hanlon K, Shapiro JI, Abraham NG. (2016). Oxidized HDL is a potent inducer of adipogenesis and causes activation of the Ang-II and 20-HETE systems in human obese females: Prostaglandins Other Lipid Mediat. 123:68-77.

21 Muñoz LJ, Riátiga D, Concha SC, (2012). Asociación de trastornos nutricionales con alteraciones del gusto y el ardor de la mucosa oral en las personas mayores residentes. Rev.UTASALUD 1(1):19-31.

22 Lee N, Duan H, Hebert MF, Liang CJ, Rice KM, Wang J. (2014). Taste of a pill: organic cation transporter-3 (OCT3) mediates metformin accumulation and secretion in salivary glands. The Journal of biological chemistry. 289(39):27055- 27064.

23 Tallis J, James RS, Seebacher F. (2018). The effects of obesity on skeletal muscle contractile function. J Exp Biol. 221: (Pt 13).

24 Roura E, Foster S, Winklebach A, Navarro M, Thomas W, Campbell K, Stowasser M. (2016). Taste and Hypertension in Humans: Targeting Cardiovascular Disease. Curr Pharm Des. 22(15):2290-305. 